\title{
Validation of FLUENT CFD model for hydrogen distribution in a containment
}

\author{
D.C. Visser ${ }^{1}$ \\ M. Houkema \\ N.B. Siccama ${ }^{1}$ \\ E.M.J. Komen ${ }^{1}$ \\ ${ }^{1}$ Nuclear Research and Consultancy Group \\ Published in: Nuclear Engineering and Design 245 (2012) pp. 161-171
}




\title{
Validation of a FLUENT CFD model for hydrogen distribution in a containment
}

\author{
D.C. Visser*, M. Houkema ${ }^{1}$, N.B. Siccama, E.M.J. Komen \\ Nuclear Research and Consultancy Group (NRG), Westerduinweg 3, 1755 ZG, Petten, The Netherlands
}

\section{A R T I C L E I N F O}

\section{Article history:}

Received 2 November 2011

Received in revised form 27 January 2012

Accepted 30 January 2012

\section{Keywords:}

Hydrogen stratification

Severe accident

CFD

Containment thermal hydraulics

\begin{abstract}
A B S T R A C T
Hydrogen may be released into the containment atmosphere of a nuclear power plant during a severe accident. Locally, high hydrogen concentrations may be reached that can possibly cause fast deflagration or even detonation and put the integrity of the containment at risk. The distribution and mixing of hydrogen is, therefore, an important safety issue for nuclear power plants.

Computational fluid dynamics (CFD) codes can be applied to predict the hydrogen distribution in the containment within the course of a hypothetical severe accident and get an estimate of the local hydrogen concentration in the various zones of the containment. In this way the risk associated with the hydrogen safety issue can be determined, and safety related measurements and procedures could be assessed. In order to further validate the CFD containment model of NRG in the context of hydrogen distribution in the containment of a nuclear power plant, the HM-2 test performed in the German THAI (thermalhydraulics, hydrogen, aerosols and iodine) facility is selected. In the first phase of the HM-2 test a stratified hydrogen-rich light gas layer was established in the upper part of the THAI containment. In the second phase steam was injected at a lower position. This induced a rising plume that gradually dissolved the stratified hydrogen-rich layer from below. Phenomena that are expected in severe accidents, like natural convection, turbulent mixing, condensation, heat transfer and distribution in different compartments, are simulated in this hypothetical severe accident scenario.

The hydrogen distribution and associated physical phenomena monitored during the HM-2 test are predicted well by the CFD containment model. Sensitivity analyses demonstrated that a mesh resolution of $45 \mathrm{~mm}$ in the bulk and $15 \mathrm{~mm}$ near the walls is sufficiently small to adequately model the hydrogen distribution and dissolution processes in the THAI HM-2 test. These analyses also showed that wall functions could be applied. Sensitivity analyses on the effect of the turbulence model and turbulence settings revealed that it is important to take the effect of buoyancy on the turbulent kinetic energy into account. When this effect of buoyancy is included, the results of the standard $k-\varepsilon$ turbulence model and SST $k-\omega$ turbulence model are similar and agree well with experiment. The outcome of these sensitivity analyses can be used as input for setting up the guidelines on the application of CFD for containment issues.
\end{abstract}

(C) 2012 Elsevier B.V. All rights reserved.

\section{Introduction}

During a severe accident in water-cooled reactors, large quantities of hydrogen and steam can be released into the containment. The hydrogen, generated as a result of core degradation and oxidation, can form a combustible gas mixture with the oxygen present in the containment atmosphere. Unintended ignition of this mixture can initiate a combustion process, which may damage relevant safety systems and challenge the integrity of the containment. In the worst-case, the safety function of the containment can get lost.

\footnotetext{
* Corresponding author. Tel.: +31 22456 4193; fax: +31224568490.

E-mail address: visser@nrg.eu (D.C. Visser).

1 Present address: Energy research Centre of the Netherlands (ECN), Petten, The Netherlands.
}

The potential danger of hydrogen was first realized after the Three Mile Island accident in 1979, where a large quantity of hydrogen was released into the containment and started burning. Since then, many efforts have been taken to mitigate and/or reduce the potential risk of hydrogen. For instance, by installation of hydrogen recombiners that convert hydrogen to steam. The recent hydrogen explosions during the Fukushima Daiichi accident in March 2011 showed, however, that the control and mitigation of the hydrogen risk is still a key safety issue for nuclear power plants.

In order to assess the potential risk of hydrogen and the effectiveness of the mitigation systems installed in the containment, it is necessary to predict the hydrogen concentration in the containment during a severe accident. Two thermal-hydraulic approaches can be used for this prediction (SOAR, 1999): the lumped parameter (LP) and the computational fluid dynamics (CFD) approach. The LP codes are of practical use because they are able to give a quick estimate on the hydrogen distribution during a severe accident. The 


\begin{tabular}{|ll|}
\hline \multicolumn{2}{|c|}{ Nomenclature } \\
$G_{b}$ & buoyancy effect source term $\left(\mathrm{kg} / \mathrm{m} \mathrm{s}^{3}\right)$ \\
$g$ & gravitational acceleration $\left(\mathrm{m} / \mathrm{s}^{2}\right)$ \\
$k$ & turbulent kinetic energy $\left(\mathrm{m}^{2} / \mathrm{s}^{2}\right)$ \\
$\mathrm{Pr}_{t}$ & turbulent Prandtl number $(-)$ \\
$\operatorname{Re}$ & Reynolds number $(-)$ \\
& \\
Greek symbols \\
$\varepsilon$ & turbulent dissipation rate $\left(\mathrm{m}^{2} / \mathrm{s}^{3}\right)$ \\
$\rho$ & density $\left(\mathrm{kg} / \mathrm{m}^{3}\right)$ \\
$\mu_{t}$ & turbulent viscosity $(\mathrm{kg} / \mathrm{ms} \mathrm{s})$ \\
$\omega$ & specific dissipation rate $(1 / \mathrm{s})$ \\
\hline
\end{tabular}

high resolution CFD codes provide detailed information on local phenomena and concentrations. The OECD launched the international benchmark exercise ISP47 in order to assess the capabilities of both approaches for containment analyses (Allelein et al., 2007). A combined use of LP and CFD was recommended, where LP will act as the main workhorse and CFD will be used for more detailed analyses with high (local) resolution.

The 3D special purpose CFD codes GOTHIC, GASFLOW and TONUS were the first CFD codes designed for containment analyses. GOTHIC is an EPRI-sponsored code that can be used for either lumped-parameter computations or for more detailed multidimensional analysis (Andreani and Paladino, 2010). GASFLOW is a joint development of Forschungszentrum Karlsruhe and Los Alamos National Laboratory for the simulation of steam/hydrogen distribution and combustion in complex nuclear reactor containment geometries (GASFLOW, 2011). TONUS is the French in-house hydrogen risk analysis code developed by CEA and IRSN (Kudriakov et al., 2008). Compared to more recent commercial CFD codes like CFX and FLUENT, these early codes are limited in their meshing capabilities, turbulence modeling and multiprocessor parallel performance. Therefore the interest and use of the new, general purpose commercial codes for containment analyses increased substantially over the last years (Heitsch et al., 2010; Prabhudharwadkar et al., 2011). The GOTHIC, GASFLOW and TONUS codes are, however, still readily employed because of their specific models (e.g. steam condensation and hydrogen mitigation) and extensive validation.

Although the CFD codes have proven to be a powerful tool, extensive validation and clear guidelines are necessary before these codes can be reliably used for real plant analyses. In the present work, the containment model developed by NRG in the commercial CFD code FLUENT is further validated using the well instrumented and well defined THAI HM-2 test. This test simulates the relevant physical phenomena involved in the context of hydrogen distribution in a large, multi-compartment containment under severe accident conditions (Kanzleiter and Fischer, 2008).

The international benchmark exercise ISP47 (Allelein et al., 2007) as well as the HM-2 benchmark exercise within the OECD-NEA THAI project (Schwarz et al., 2010) showed a strong user-dependence, which demonstrates the importance of setting up and applying best practice guidelines (BPGs) specific for containment applications. To make a start with the development of such BPGs, sensitivity analyses are performed in the present work to analyse the effect of mesh resolution, near-wall treatment, turbulence modeling and turbulence settings on the hydrogen distribution in a containment.

The work presented here is part of NRG's program on the long term development and validation of a reliable and complete CFD containment model for hydrogen distribution and combustion, including mitigation systems such as recombiners, sprays, and condensers.

\section{THAI HM-2 experiment}

The THAI facility is operated by Becker Technologies in Eschborn, Germany. The objective of the HM (hydrogen mixing) test series within the OECD-NEA THAI project was to study hydrogen mixing and distribution in a large, multi-compartment containment. Test HM-1 was performed with inert helium gas and test HM-2 with hydrogen gas. A detailed description and comparison of the HM tests is given by Gupta et al. (2010). The configuration of the THAI facility, instrumentation and test conditions of the HM-2 experiment are specified by Kanzleiter and Fischer (2008).

Fig. 1 shows the THAI vessel with the internal structures as employed in the HM tests. The THAI vessel is a cylindrical containment with a height of $9.2 \mathrm{~m}$, a diameter of $3.2 \mathrm{~m}$ and a total volume of $60 \mathrm{~m}^{3}$. The HM test setup facilitates the study of hydrogen mixing in a multi-compartment facility: the vessel contains an inner cylinder and four condensate trays that divide the vessel into a base, a cylinder, an annulus and a dome region. These different compartments in the THAI vessel are indicated in the schematic drawing in Fig. 1. The inner cylinder with a height of $4 \mathrm{~m}$ and a diameter of $1.4 \mathrm{~m}$ is open at both ends. The condensate trays at an elevation of $4 \mathrm{~m}$ from the bottom of the vessel block $2 / 3$ of the cross-sectional area in the annulus. Various instrumentation devices are installed at different locations in the vessel for measuring the hydrogen concentration, temperature, pressure and flow velocity.

At the start of the HM-2 test, the vessel atmosphere consists of $98 \mathrm{vol} \%$ nitrogen gas, $1 \mathrm{vol} \%$ oxygen and 1 vol\% steam at ambient conditions ( 1 bar, $21^{\circ} \mathrm{C}$ ). The HM-2 test can be divided in two phases;

- Phase-1: hydrogen/steam injection and formation of a stable stratified hydrogen-rich gas layer in the upper part of the vessel (0-4300 s).

- Phase-2: steam injection, dissolution of the stratified hydrogenrich gas layer and mixing of the atmosphere in the vessel (4300-6860s).

In phase-1, a mixture of hydrogen $(\sim 0.3 \mathrm{~g} / \mathrm{s})$ and saturated steam $(\sim 0.24 \mathrm{~g} / \mathrm{s})$ is injected in upward direction into the annulus from a circular pipe of $28.5 \mathrm{~mm}$ diameter at an elevation of $4.8 \mathrm{~m}$. The average injection temperature during this phase is $45^{\circ} \mathrm{C}$. At the end of phase-1, from 4200 to 4300 s, there is no injection. In phase-2, saturated steam $(\sim 24 \mathrm{~g} / \mathrm{s})$ is injected in upward direction below the centre of the inner cylinder from a nozzle of $138 \mathrm{~mm}$ diameter at an elevation of $1.8 \mathrm{~m}$. The average injection temperature during phase- 2 is $108^{\circ} \mathrm{C}$. Detailed injection rates and injection temperatures during phase- 1 and phase- 2 of the HM- 2 test are shown in Fig. 2. Physical phenomena like convection, turbulent mixing, condensation, heat transfer and distribution of gasses into different compartmens are simulated in these phases of the HM-2 test. These phenomena can be very relevant in the course of a severe accident.

\section{CFD model}

\subsection{Introduction}

The transient calculations are performed with FLUENT, a commercial general-purpose CFD package supplied by ANSYS Inc. In all calculations, one half of the THAI vessel is modeled, assuming symmetry across the vertical plane through the vessel axis. Since isotropic Reynolds averaged Navier-Stokes (RANS) 

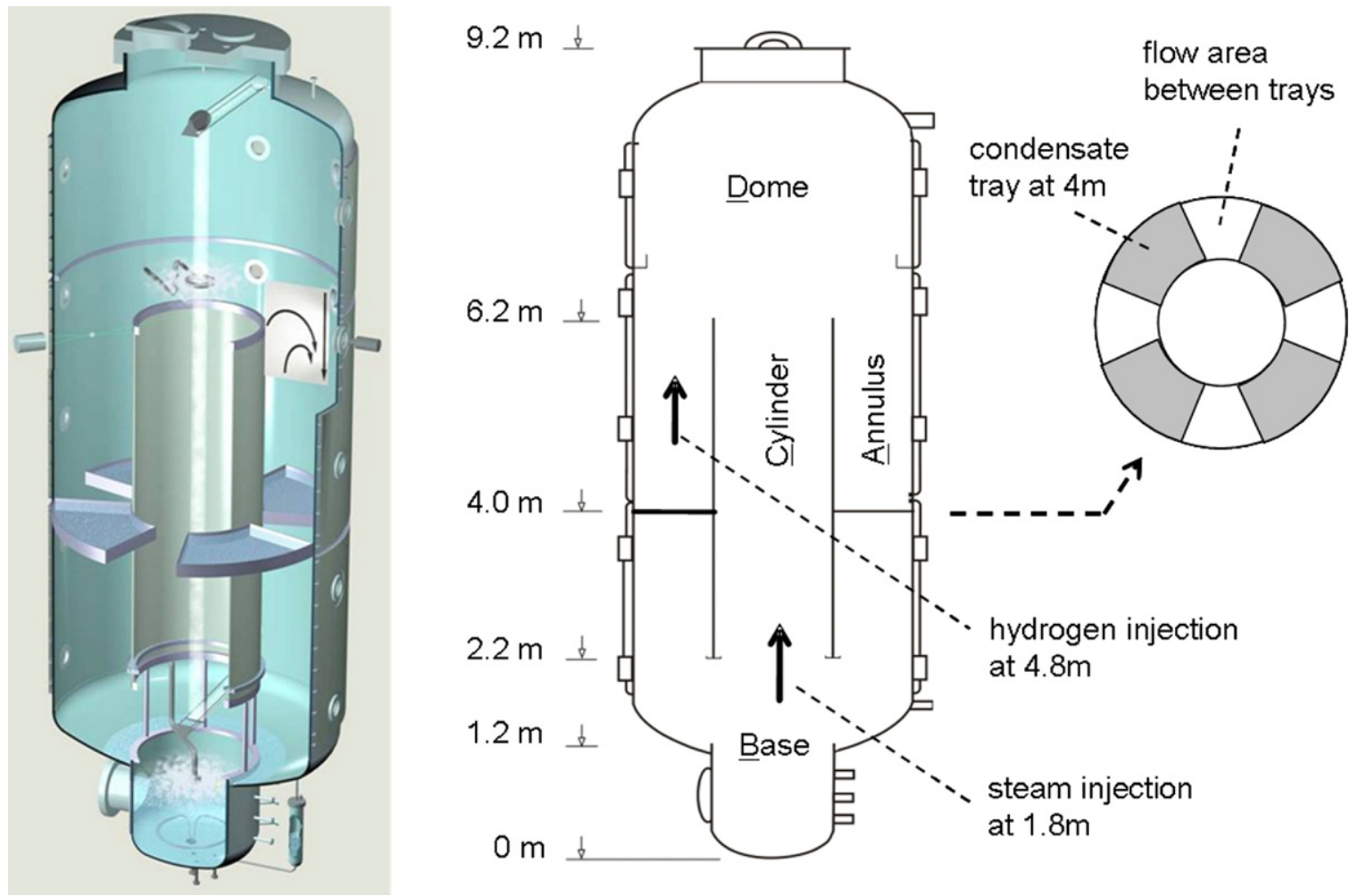

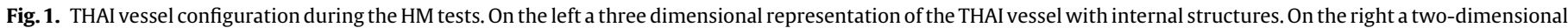
representation showing the compartments and injection points.
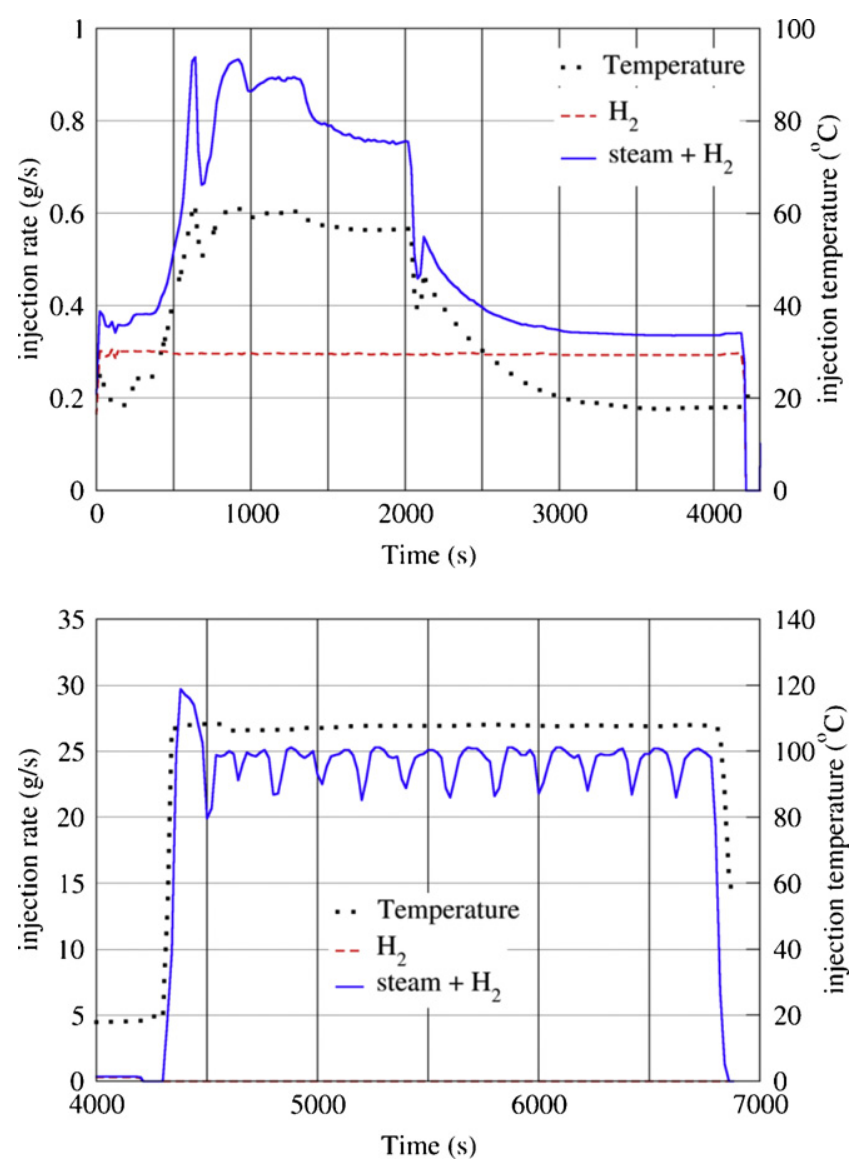

Fig. 2. Injection rates and injection temperatures during phase-1 (top) and phase-2 (bottom) of the THAI HM-2 test (Kanzleiter and Fischer, 2008). turbulence models are applied, it is expected that this geometrical simplification have no effect as has been verified by Royl et al. (2009). The modeled three-dimensional geometry is shown in Fig. 3.

The solid walls and solid internal structures of the THAI vessel are modeled to take into account the effect of heat conduction and heat capacity. Geometrical information and thermal properties of the solids are taken from Fischer (2004). The fluid in the vessel is modeled as a composition and temperature dependent ideal

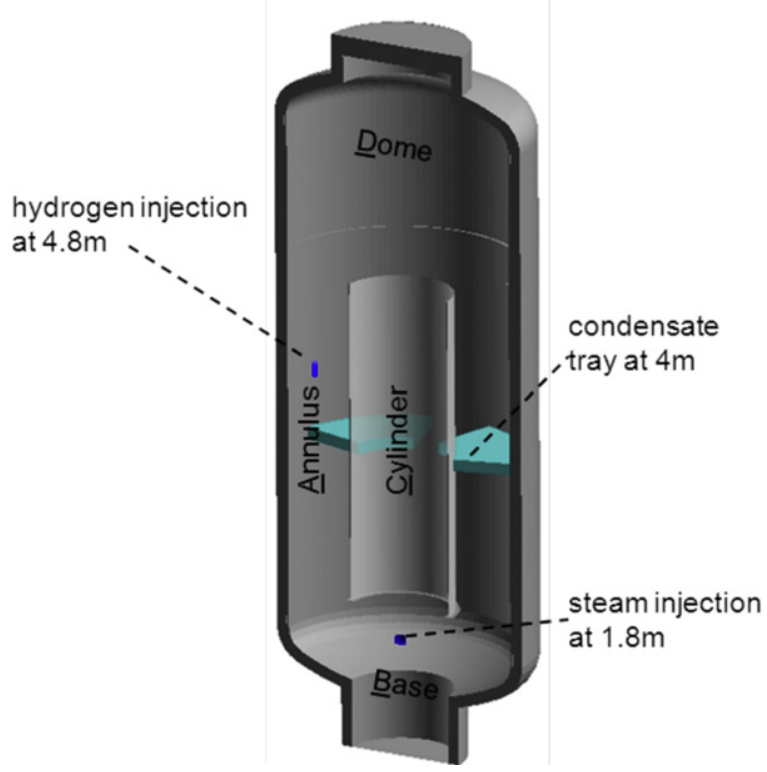

Fig. 3. Three-dimensional geometry applied in the CFD calculations; one symmetrical half of the THAI vessel. 
Table 1

General features of the CFD model.

\begin{tabular}{ll}
\hline Code version & FLUENT 6.3 \\
\hline Solver & Pressure-based segregated \\
Formulation & Transient \\
Turbulence approach & RANS \\
Pressure interpolation scheme & Body-force weighted \\
Pressure correction scheme & PISO \\
Spatial discretization & 2nd Order upwind \\
Temporal discretization & 2nd Order implicit \\
Geometry & 3-Dimensional, half vessel \\
Walls & No slip \\
Near-wall treatment & Enhanced wall treatment \\
Conduction & 3-dimensional \\
Fluid properties & Composition and temperature \\
& dependent ideal gas \\
Condensation & User-defined function, NRG \\
Time step size & condensation model \\
& Increases from $0.1 \mathrm{~s}$ (at the start of \\
& each phase) to 0.2 s (after $20 \mathrm{~s}$ from the \\
& start of each phase)
\end{tabular}

gas mixture of its constituent components (nitrogen, hydrogen and steam). The temperature dependence of specific heat, thermal conductivity and viscosity is implemented by means of a piecewiselinear approach for each of the individual gas components. The temperature dependent data for nitrogen, hydrogen and steam are obtained from Lemmon et al. (2007). Also the diffusion coefficients of the components depend on gas composition and temperature. The effect of the $1 \mathrm{vol} \%$ oxygen initially present is neglected.

In general, the guidelines given in the FLUENT 6.3 User's Guide (2006) and by ERCOFTAC (2000) are followed for setting up the CFD model. An overview of the general features of the applied CFD model is given in Table 1. A condensation model was developed and used by NRG, since there are no models for wall and bulk condensation available in FLUENT. These condensation processes are incorporated in the CFD model by means of user-defined functions (UDF) referred to as the NRG Condensation Model. The NRG Condensation Model, the initial and boundary conditions as well as the applied meshes and turbulence models are described in the following subsections.

\section{2. $N R G$ condensation model}

The implemented NRG Condensation Model takes into account the following processes:

1. Bulk condensation and evaporation;

2. Wall condensation;

3. Deposition of bulk condensate on walls;

4. Rainout of bulk condensate.

The condensation/evaporation process in the bulk and at the wall is modeled by the reaction;

$\mathrm{H}_{2} \mathrm{O}(g) \stackrel{k_{r}}{\longleftrightarrow} \mathrm{H}_{2} \mathrm{O}(l)+$ heat

where the reaction rate $k_{\mathrm{r}}$ is controlled by the vapor pressure. Evaporation of condensate from the walls is not expected and not taken into account. Furthermore, the water condensate that is deposited on the walls or rains out from the bulk is not treated in the model. The effect of this water condensate on for instance the flow, heat transfer and condensation is thus neglected. The NRG condensation model is described in more detail by Houkema et al. (2008) and has been employed successfully in the SARNET Condensation Benchmark (Ambrosini et al., 2007) and International Standard Problem ISP-47 on Containment Thermalhydraulics (Allelein et al., 2007).
Table 2

Initial conditions for the CFD calculations.

\begin{tabular}{ll}
\hline Pressure & $1.008 \mathrm{bar}$ \\
\hline Temperature & Linear temperature increase from \\
& $18.6^{\circ} \mathrm{C}$ at the bottom to $23.3^{\circ} \mathrm{C}$ at \\
& the top of the THAI vessel. \\
& $99 \mathrm{vol} \%$ nitrogen $(1 \mathrm{vol} \%$ oxygen is \\
Composition & neglected $1 \mathrm{vol} \%$ steam \\
Velocity & $\mathrm{u}, \mathrm{v}, \mathrm{w}=0 \mathrm{~m} / \mathrm{s}$ (fluid initially at rest) \\
Turbulence & $k, \varepsilon=10^{-6}($ low turbulence \\
& assumed $)$ \\
\hline
\end{tabular}

\subsection{Initial and boundary conditions}

The initial conditions for the THAI HM-2 test are specified by Kanzleiter and Fischer (2008) and are adopted as starting point of phase- 1 in the calculations. The applied initial conditions are listed in Table 2, where it must be noted that the linear temperature increase is applied for the solid walls as well.

Hydrogen and steam injection is modeled with mass-flow inlet boundary conditions. The injection rates and injection temperatures during phase- 1 and phase- 2 of the HM- 2 test are specified by Kanzleiter and Fischer (2008) and are shown in Fig. 2. These time dependent boundary conditions are prescribed in tabular form in the FLUENT CFD code. The turbulence quantities at the inlets are specified in terms of turbulence intensity $(I)$ and hydraulic diameter $\left(D_{\mathrm{h}}\right)$.

No-slip boundary conditions are imposed on the solid walls and solid structures, using the enhanced wall treatment (EWT) approach in FLUENT to model the flow near the walls. The EWT is a near-wall modeling method that combines a two-layer model with (enhanced) wall functions. If the near-wall mesh is fine enough (typically $y^{+} \approx 1$ ), the EWT will automatically resolve the laminar sublayer. In all other cases, the EWT will automatically make use of wall-functions. A similar approach is followed for modeling the near-wall heat and species transport. The vessel's outer wall is insulated and assumed adiabatic. All other walls are modeled as fluid-solid interfaces with conjugate heat-transfer. Heat transfer by means of radiation is neglected. Condensation takes place on all the walls that are in contact with the gas mixture inside the vessel.

\subsection{Computational mesh}

Four different computational meshes are constructed in order to study the effect of mesh resolution and near-wall treatment (twolayer model or wall-functions). The four meshes are constructed in a similar way. Table 3 presents the characteristics of the four meshes. Fig. 4 shows the "standard" mesh. The solid regions are filled with hexahedral cells. The fluid regions consist of a hybrid mesh with tetrahedral and hexahedral cells. The vessel's base region is mostly filled with unstructured tetrahedral mesh cells. The annulus, cylinder and dome region consist of structured and unstructured hexahedral cells. The mesh is refined towards the walls and solid structures in order to resolve the flow and physical phenomena near the wall in more detail. The mesh at and above the inlets is refined in order to resolve the small inflow area and the injection jet in more detail.

The characteristics of the four meshes are listed in Table 3. In the $y^{+}=1$ mesh, the typical cell size is $0.25 \mathrm{~mm}$ near the walls and $30 \mathrm{~mm} \times 50 \mathrm{~mm}$ in the bulk. In the standard, coarse and fine mesh the typical cell size is $15 \mathrm{~mm}$ near the walls and $30 \mathrm{~mm} \times 50 \mathrm{~mm}$, $45 \mathrm{~mm} \times 75 \mathrm{~mm}$ and $20 \mathrm{~mm} \times 30 \mathrm{~mm}$ in the bulk, respectively Fig. 5 compares the four meshes at a section near the inner cylinder. Depending on the mesh resolution and the flow properties near the wall, the EWT approach in FLUENT makes use of wall functions or resolves the viscous boundary layer at the wall. The small 
Table 3

Constructed computational meshes.

\begin{tabular}{|c|c|c|c|c|}
\hline Mesh & $y^{+}=1$ mesh & Standard mesh & Coarse mesh & Fine mesh \\
\hline Total number of cells & 763.905 & 543.438 & 175.069 & 2562.528 \\
\hline Number of fluid cells & 671.225 & 453.158 & 139.160 & 2197.401 \\
\hline Typical cell size in the bulk (radial $\times$ vertical direction) & $30 \mathrm{~mm} \times 50 \mathrm{~mm}$ & $30 \mathrm{~mm} \times 50 \mathrm{~mm}$ & $45 \mathrm{~mm} \times 75 \mathrm{~mm}$ & $20 \mathrm{~mm} \times 30 \mathrm{~mm}$ \\
\hline Typical cell size at the wall & $0.25 \mathrm{~mm}$ & $15 \mathrm{~mm}$ & $15 \mathrm{~mm}$ & $15 \mathrm{~mm}$ \\
\hline Typical $y^{+}$ & $\leq 1$ & $5-20$ & $5-20$ & $5-20$ \\
\hline Near-wall treatment & Two-layer model & Wall functions & Wall functions & Wall functions \\
\hline
\end{tabular}
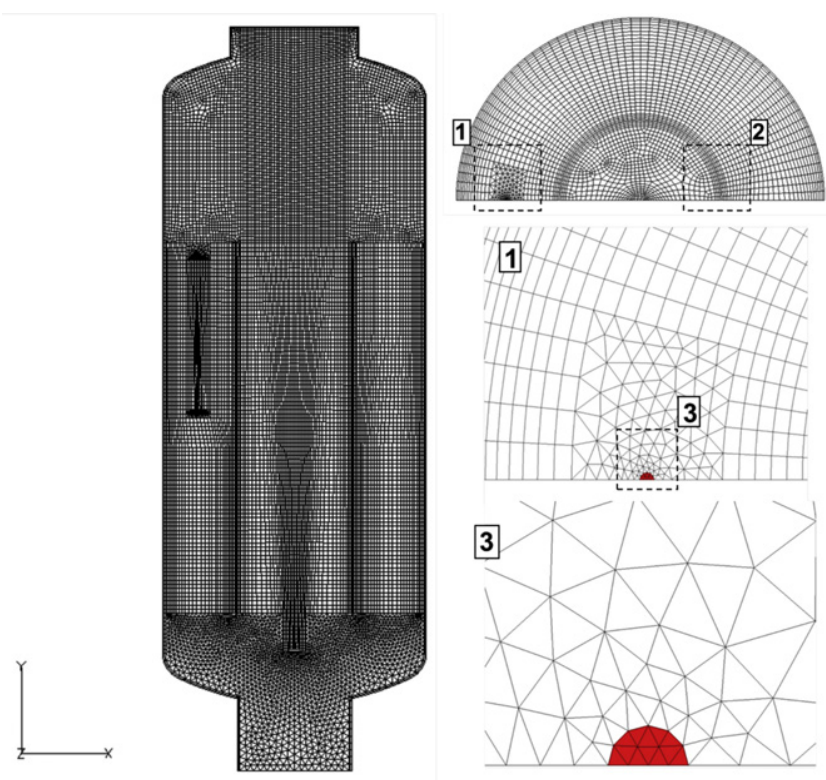

Fig. 4. Front view of the standard mesh on the vessel's symmetry plane (left) and top view of the standard mesh on the horizontal cross section through the hydrogen inlet at $y=4.8 \mathrm{~m}$ (right). The mesh at the hydrogen inlet is shown in more detail for Sections 1 and 3. The inflow area is filled/colored. The mesh resolution near the wall of the inner cylinder (Section 2) is shown in more detail for the four different meshes in Fig. 5.

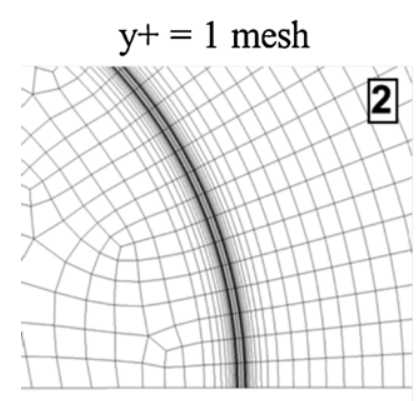

coarse mesh

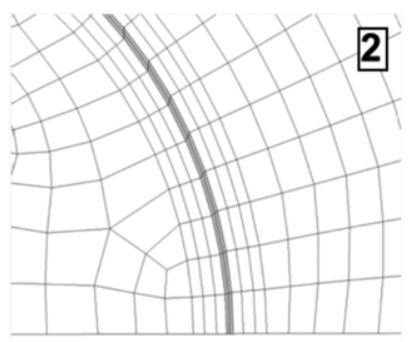

standard mesh

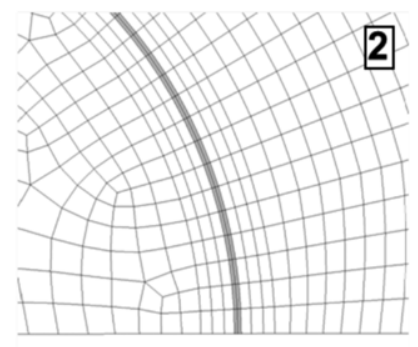

fine mesh

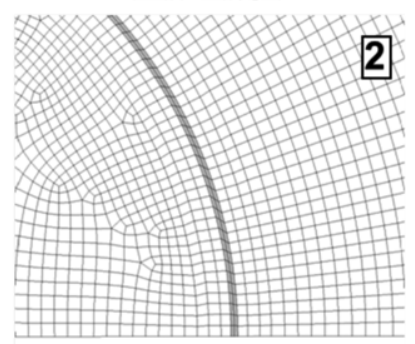

Fig. 5. Top view of the four constructed meshes at the wall of the inner cylinder (Section 2 in Fig. 4).
Table 4

Considered turbulence settings.

\begin{tabular}{lll}
\hline Case & $\begin{array}{l}\text { Turbulence } \\
\text { model }\end{array}$ & Buoyancy effect included in \\
\hline 1 (reference case) & SKE & $k$ and ( full buoyancy option) \\
2 & SKE & $k$ (by default) \\
3 & SKW & None (by default) \\
4 & SKW & $k$ (by UDF) \\
5 & SSTKW & None (by default) \\
6 & SSTKW & $k$ (by UDF) \\
\hline
\end{tabular}

near-wall cells in the $y^{+}=1$ mesh make it possible to resolve the viscous boundary layer near the walls $\left(y^{+} \leq 1\right)$. Wall functions will be applied in most near-wall regions for the standard, coarse and fine mesh $\left(y^{+}>1\right)$.

\subsection{Turbulence model}

In general, the standard $k-\varepsilon$ turbulence model (SKE) with full buoyancy effects and default turbulent constants is utilized for the CFD analyses in this paper (i.e. the reference case). In order to study the effect of the turbulence model and the buoyancy effects, the calculation for phase- 2 of the HM-2 test is repeated using the standard $k-\omega$ (SKW) and the SST $k-\omega$ turbulence model (SSTKW) with and without taking into account the effect of buoyancy on turbulence. An overview of the considered cases is given in Table 4 . This sensitivity study on turbulence settings is performed on the coarse mesh. It will be demonstrated in the next chapter that the resolution of the coarse mesh suffices to capture the relevant flow phenomena in the HM-2 test.

The $k-\varepsilon$ models as well as the $k-\omega$ models belong to the class of two-equation RANS turbulence models. In the $k-\varepsilon$ models turbulence is modeled with the transport equation for the turbulent kinetic energy $(k)$ and its dissipation rate $(\varepsilon)$. In the $k-\omega$ models turbulence is modeled with the transport equation for the turbulent kinetic energy $(k)$ and the specific dissipation rate $(\omega)$. The standard $k-\omega$ model in FLUENT is based on the Wilcox $k-\omega$ model. The SST $k-\omega$ model is developed by Menter (1994) and combines the best of the $k-\varepsilon$ and $k-\omega$ formulations, blending the robust and accurate $k-\omega$ formulation in the near-wall region with the reliable $k-\varepsilon$ formulation in the bulk region. A detailed description of the $k-\varepsilon$ and $k-\omega$ turbulence models is given in the FLUENT 6.3 User's Guide (2006).

In a non-zero gravity field, buoyancy forces can suppress or promote turbulence in the presence of density gradients. Buoyancy tends to suppress turbulence at a stable stratification and buoyancy promotes turbulence at an unstable stratification. The production (or dissipation) of turbulence by buoyancy can be incorporated in the $k-\varepsilon$ and $k$ - $\omega$ turbulence models by adding the source term $G_{b}$ to the transport equation for $k$. The source term $G_{b}$ is defined as

$G_{b}=-g_{y} \frac{\mu_{t}}{\rho \operatorname{Pr}_{t}} \frac{\partial \rho}{\partial y}$,

where $y$ is the vertical direction, $g_{y}$ the gravitational acceleration in the $y$-direction, $\mu_{t}$ the turbulent viscosity, $\rho$ the density and $\operatorname{Pr}_{t}$ the turbulent Prandtl number. In FLUENT, $G_{b}$ is only included by default in the $k$-equation of all the $k-\varepsilon$ models. It is however possible 
to incorporate $G_{b}$ in the $k$-equation of the $k$ - $\omega$ models as well by means of a 'so called' user-defined function (UDF).

Since the effect of buoyancy on the turbulent dissipation rate $\varepsilon$ is not well understood, by default this effect is not included in the transport equation for $\varepsilon$ in FLUENT (FLUENT, 2006). A certain effect of buoyancy on $\varepsilon$ can be included in the $k-\varepsilon$ models of FLUENT by activating the "full buoyancy" option. The effect of buoyancy on $\varepsilon$ can be observed by comparison of cases 1 and 2 in Table 4 . The effect of buoyancy is not included in the $\omega$-equation of the $k$ - $\omega$ models in FLUENT and is therefore not considered here.

\section{Results}

In this chapter, the results of the CFD analyses are presented and compared to the HM-2 experiment performed by Becker Technologies (EXPBT). The results of the sensitivity analyses on mesh resolution and turbulence models/settings are presented here as well. This chapter is divided into the following sections:

Section 4.1: comparison of the experimental and CFD results for phase-1.

Section 4.2: comparison of the experimental and CFD results for phase-2.

Section 4.3: effect of mesh resolution and near-wall treatment. Section 4.4: effect of turbulence model and buoyancy effects.

The CFD results presented in Sections 4.1 and 4.2 are obtained with the standard $k-\varepsilon$ turbulence model (SKE) with full buoyancy effects on the $y^{+}=1$ mesh.

\subsection{Phase -1 results $(0-4300 \mathrm{~s})$}

In phase- 1 of the HM-2 test, a total amount of $1.24 \mathrm{~kg}$ hydrogen and $1 \mathrm{~kg}$ saturated steam is injected into the THAI vessel from a vertical pipe in the annulus. Since the THAI vessel is closed, the hydrogen and steam content will increase, and therewith the pressure as well. The measured and calculated evolution of the atmospheric pressure and hydrogen mass in the vessel during phase- 1 is shown in Fig. 6. The CFD calculation shows an accurate mass balance and a negligible error of less than $0.2 \%$ in the amount of hydrogen in the system. The calculation shows, however, a slight over-prediction of the vessel pressure (about 0.02 bars at the end of phase-1).

The injected gas mixture of hydrogen and steam has a relatively low density and forms a stable stratified hydrogen-rich gas layer in the upper part of the THAI vessel. Fig. 7 shows the hydrogen distribution at the end of phase- 1 on a vertical line through the annulus. The experiment and calculation show a similar hydrogen distribution in the THAI vessel. Initially the vessel contains no hydrogen. At the end of phase-1, a hydrogen-rich gas layer is formed in the upper half of the vessel, while the hydrogen concentration remains below $1 \%$ in the lower half of the vessel. A strong gradient in hydrogen concentration is observed at an elevation of $4-5 \mathrm{~m}$. The inset in Fig. 7 is a contour plot of the predicted hydrogen concentration on the symmetry plane at $4300 \mathrm{~s}$. This contour clearly shows that the hydrogen accumulates and is well mixed in the upper half of the vessel. The measured and predicted values of the hydrogen concentration in the hydrogen-rich gas layer differ slightly. In the experiment, concentrations up to $37 \mathrm{vol} \%$ are found in the upper part of the vessel, against concentrations up to $35.5 \mathrm{vol} \%$ in the calculation.

During phase-1, a mixture of hydrogen and saturated steam is injected at an average temperature of $45^{\circ} \mathrm{C}$. The temperature of the gas and the solid structures in the vessel is lower, which causes about $50 \%$ of the injected steam to condensate in the CFD calculation. Condensation of steam lowers the pressure in the vessel and
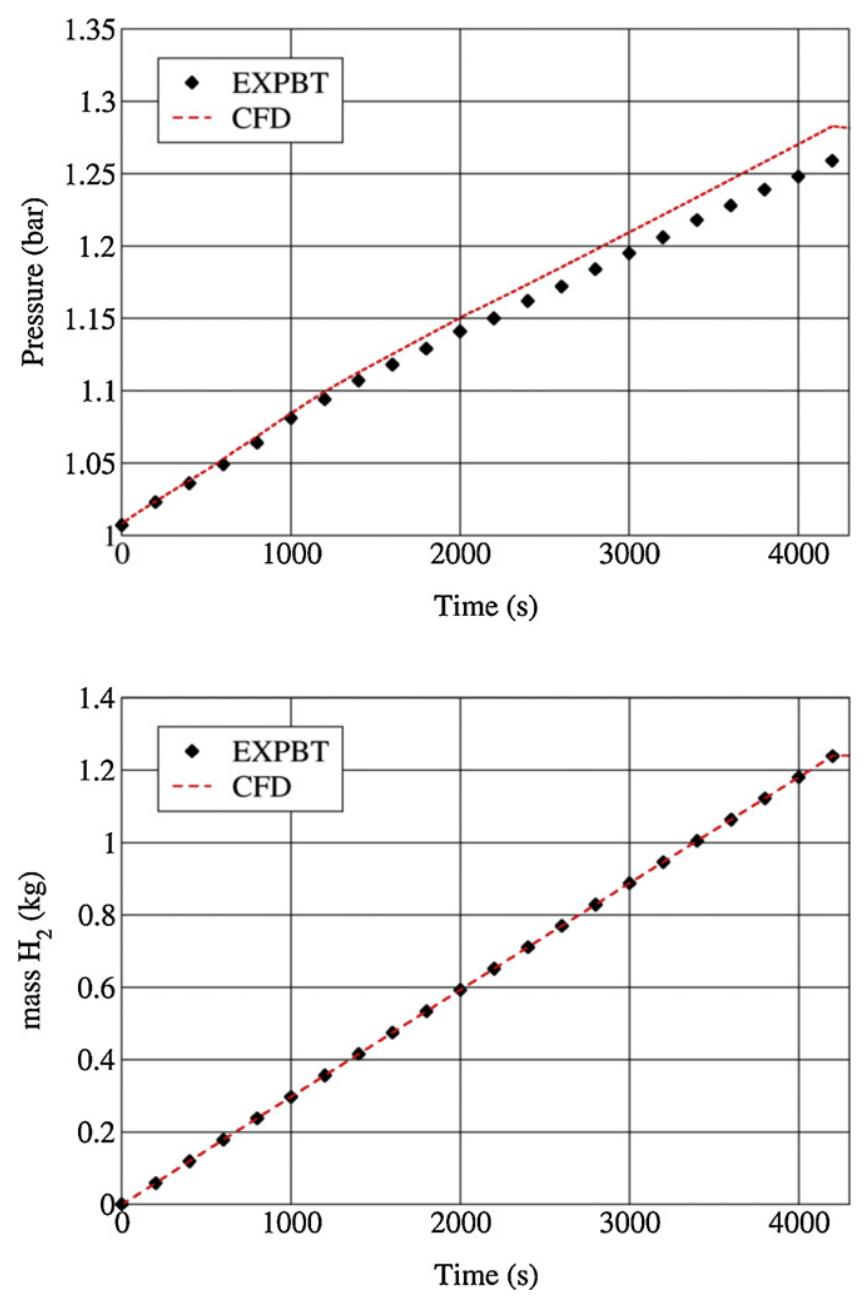

Fig. 6. Comparison of the measured (EXPBT) and predicted (CFD) atmospheric pressure (top) and amount of hydrogen (bottom) in the vessel during phase- 1 .

increases the concentration of the other species in the gas mixture. Therefore, under-prediction of condensation could be a possible reason for the observed deviation in pressure and hydrogen concentration between the CFD calculation and the experiment. This

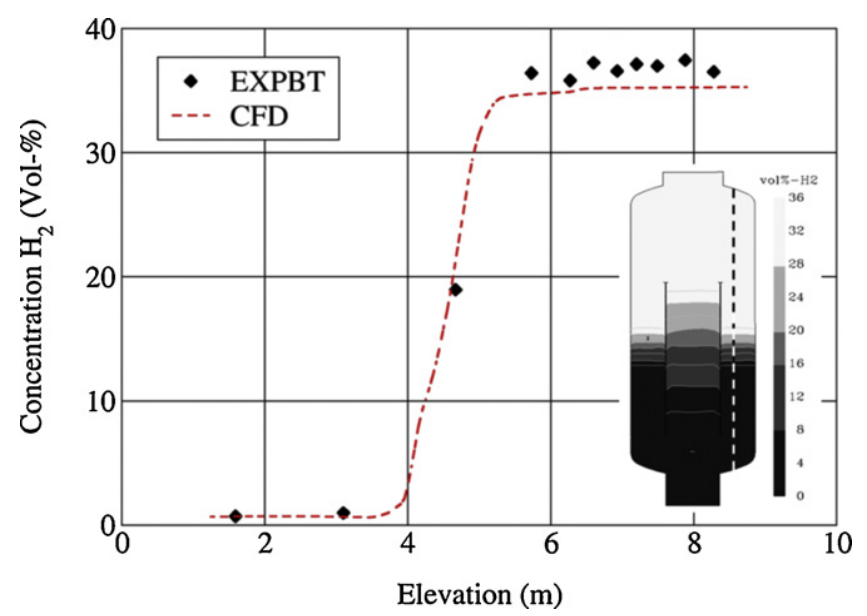

Fig. 7. Comparison of the measured (EXPBT) and predicted (CFD) hydrogen concentration at the end of phase- 1 on a vertical line running through the annulus from the bottom to the top of the THAI vessel (see dashed line in inset). The inset shows a contour plot of the predicted hydrogen concentration on the symmetry plane at the end of phase- 1 . 
effect of condensation and its importance in the THAI HM-2 test is also demonstrated by Royl et al. (2009). Under-prediction of condensation can have different causes, for instance the implemented condensation model, the imposed initial conditions (gas composition and temperature distribution) and/or the modeled heat transfer to the solids. The influence of the heat losses to the solids for the THAI HM-2 test is also considered by Schwarz et al. (2010) and Bentaib and Bleyer (2011). This heat transfer effect can be understood by observing the temperatures of the gas mixture in the vessel. The predicted gas temperature in the hydrogen-rich layer in the upper part of the vessel is about $1{ }^{\circ} \mathrm{C}$ higher than measured in the experiment, which indicates that heat transfer from the gas to the solids is slightly under-predicted. At higher temperature, less steam will condensate from the humid, hydrogen-rich layer. At the end of phase-1, the predicted steam concentration in the hydrogen-rich layer is still around $2 \mathrm{vol} \%$. Since limited experimental data is available on the steam distribution in the vessel, a direct comparison to the measured steam concentrations cannot be made and no conclusive explanation can be given for the observed differences in pressure and hydrogen concentration between calculation and experiment.

\subsection{Phase- 2 results (4300-6860s)}

In phase-2 of the HM-2 test, saturated steam at relatively high temperature is injected into the THAI vessel from a nozzle below the centre of the inner cylinder. The injected steam first clears the inner cylinder and then starts to dissolve the stable hydrogen-rich stratification in the vessel dome from below. These two consecutive processes divide phase- 2 into a stagnation and a natural circulation period. The point in time where this "natural circulation period" starts is referred to as the onset of natural circulation. During phase- 2 the amount of steam in the vessel, as predicted by the CFD calculation, increases from $1 \mathrm{~kg}$ at $4300 \mathrm{~s}$ to $6 \mathrm{~kg}$ at $6500 \mathrm{~s}$. At $6500 \mathrm{~s}$ the amount of steam injected is $\sim 53 \mathrm{~kg}$, which means that over $90 \%$ of the injected steam condensates. Condensation of steam is, thus, very important during phase- 2 .

The predicted atmospheric pressure and flow velocity above the centre of the inner cylinder during phase-2 are compared to the experimental results in Fig. 8. A vertical dashed line is drawn in the figures at $4300 \mathrm{~s}$ and at $4820 \mathrm{~s}$, which indicate the start of phase- 2 and the onset of natural circulation as determined from experiment, respectively. The period from $4300 \mathrm{~s}$ to $4820 \mathrm{~s}$ is the stagnation period. The period after $4820 \mathrm{~s}$ is the natural circulation period.

Fig. 8 shows that the measured and predicted evolution of pressure follow the same trend. The over-prediction in pressure at the end of phase- 1 remains more or less constant during phase2. Fig. 8 also compares the vertical flow velocity above the centre of the inner cylinder during phase-2. The measured and predicted flow velocity agree well on average, and fluctuations have about the same amplitude. The start of the natural circulation period is evident by the sudden significant increase in flow velocity around $4800 \mathrm{~s}$. When the onset of natural circulation is determined as the time when the flow velocity above the inner cylinder stays above $0.15 \mathrm{~m} / \mathrm{s}$, the onset found from experiment and CFD is $4820 \mathrm{~s}$ and $4720 \mathrm{~s}$, respectively. The earlier onset of natural circulation in the CFD calculation are expected to be the result of the slightly different starting conditions for phase-2 compared to the experiment. A second increase in flow velocity is observed around $6000 \mathrm{~s}$ when the hydrogen-rich gas layer is almost completely dissolved. The velocity drops to zero when the injection of steam is stopped.

Fig. 9 compares the hydrogen concentration during phase- 2 at four different locations in the vessel. Each location is in a different compartment of the vessel (base, annulus, cylinder and dome) as shown in the insets in Fig. 9. Fig. 10 shows snapshots of the
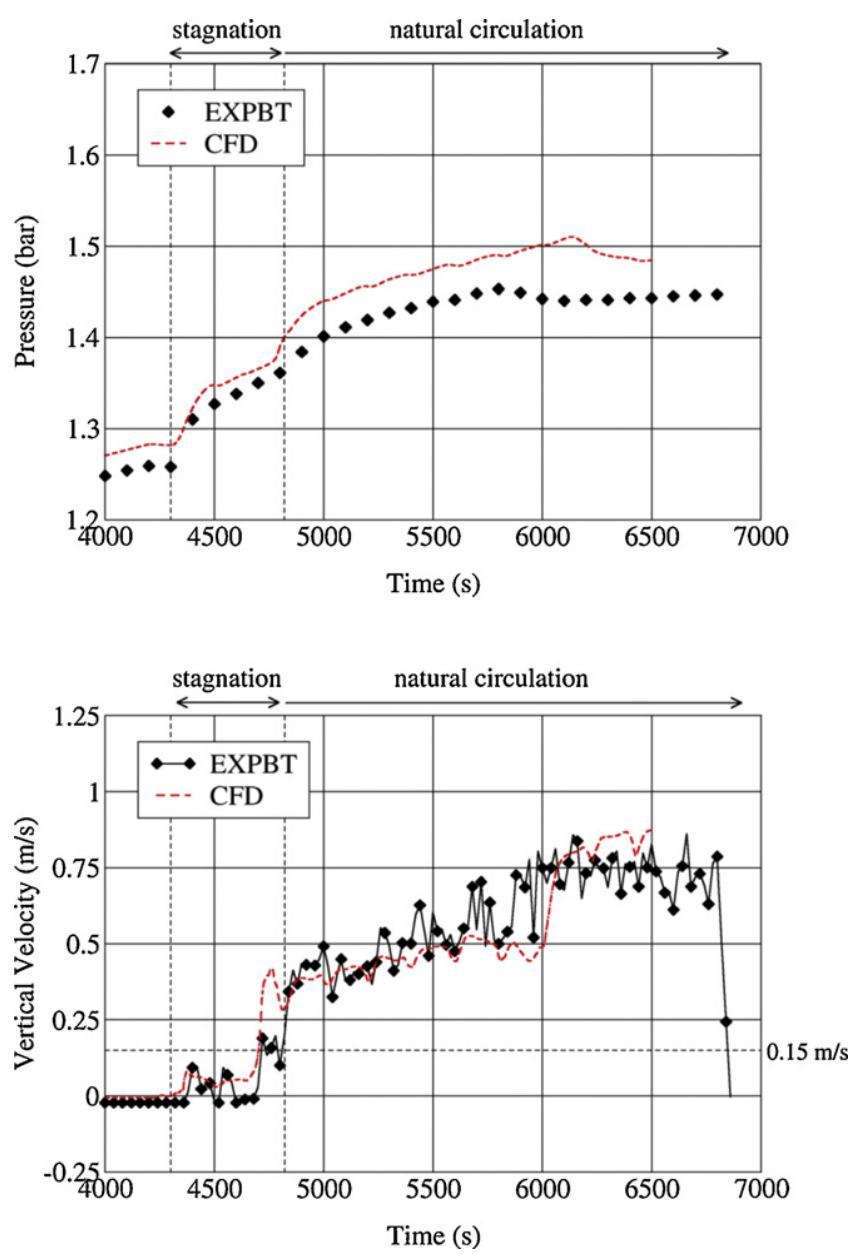

Fig. 8. Atmospheric pressure (top) and vertical flow velocity above the centre of the inner cylinder (bottom) during phase-2. The experiment ends at $6860 \mathrm{~s}$, the calculation runs up to $6500 \mathrm{~s}$.

predicted hydrogen distribution and flow field on the symmetry plane at 4300 s, $4500 \mathrm{~s}$ and $5000 \mathrm{~s}$. These snapshots are helpful to illustrate and understand the processes during phase-2. As shown in Figs. 9 and 10, the hydrogen concentration in the upper part of the vessel is uniformly high at the start of phase- 2 , while it is

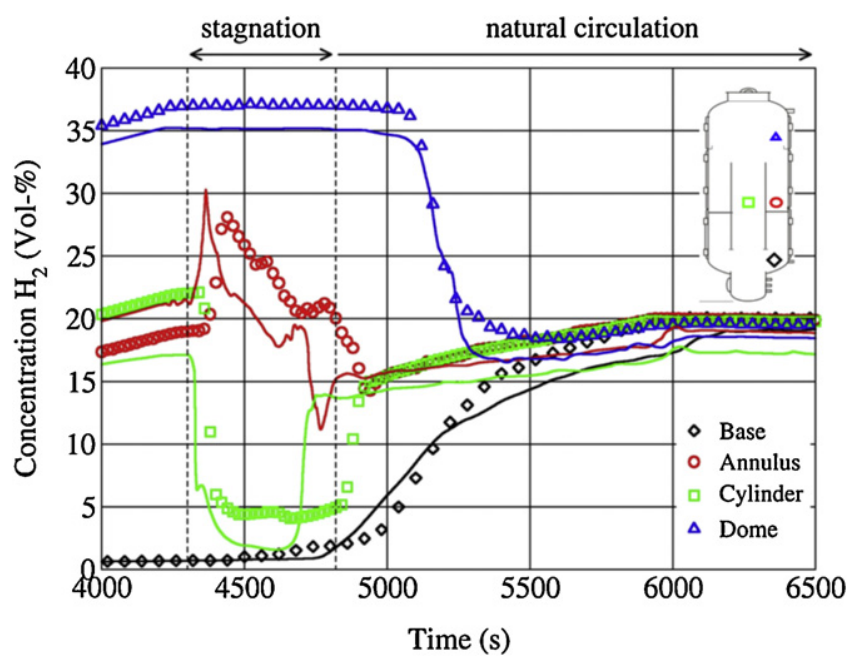

Fig. 9. Hydrogen concentrations in the vessel during phase-2 as measured (symbols) and computed (solid lines) for the THAI HM-2 test. 
Table 5

Effect of mesh resolution.

\begin{tabular}{|c|c|c|c|c|}
\hline Mesh & $y^{+}=1$ mesh & Standard mesh & Coarse mesh & Fine mesh \\
\hline Pressure at $4300 \mathrm{~s} / 5000 \mathrm{~s}$ (bar) & $1.281 / 1.461$ & $1.277 / 1.436$ & $1.278 / 1.437$ & $1.276 / 1.449$ \\
\hline Max. $\mathrm{H}_{2}$ concentration at $4300 \mathrm{~s}(\mathrm{vol} \%)$ & 35.33 & 35.61 & 35.46 & 35.56 \\
\hline Amount of $\mathrm{H}_{2}$ at $4300 \mathrm{~s}(\mathrm{~kg})$ & 1.232 & 1.243 & 1.242 & 1.241 \\
\hline Onset of natural circulation (s) & 4720 & 4780 & 4790 & 4770 \\
\hline
\end{tabular}

uniformly low in the lower part of the vessel. An interface is present in-between these two regions where the hydrogen concentration varies.

In the stagnation period, the upper end of the inner cylinder is blocked by the light gas cloud in the vessel dome and most of the injected hot steam remains in the inner cylinder, quickly displacing and diluting the gas mixture in the inner cylinder. This process can be observed in Fig. 9 by the sharp decrease of hydrogen concentration in the cylinder just after the start of phase-2. The snapshot at $4500 \mathrm{~s}$ in Fig. 10 clearly shows that the steam circulates in the inner cylinder and displaces the hydrogen-rich gas mixture initially present. It can also be observed from this snapshot that small portions of the steam-rich gas mixture spill over the upper edge of the inner cylinder and flow down along the outside of the cylinder wall. This process mixes the atmosphere in the annulus in downward direction as shown in Fig. 9 by the gradual decrease of hydrogen concentration in the annulus. The concentration in the upper (dome) and bottom (base) part of the vessel stay almost unaffected during the stagnation period. All these trends are predicted well by the CFD analysis.

Once the atmosphere in the annulus is mixed down to the lower edge of the inner cylinder, the steam-rich atmosphere in the annulus and the inner cylinder connect, forming a closed loop. This is the start of the natural circulation period where a circulating flow sets in that, driven by the steam injection, moves up through the inner cylinder and down through the annulus as illustrated by the snapshot at $5000 \mathrm{~s}$ in Fig. 10. The atmosphere in the inner cylinder and annulus is quickly mixed after the onset of this convection loop, averaging out the hydrogen concentrations in these regions. This process can be clearly observed in Fig. 9 by the sharp rise of hydrogen concentration in the cylinder around $4820 \mathrm{~s}$ for the experiment and $4720 \mathrm{~s}$ for the CFD analysis. The convection loop through and out of the inner cylinder starts to dissolve the stratified hydrogenrich gas layer from below and homogenize the atmosphere in the vessel. This is observed in Fig. 9 by the drop of hydrogen concentration in the dome of the vessel around 5200 s. Again, these trends are predicted well with CFD.

\subsection{The effect of mesh resolution and near-wall treatment}

In this section, the effect of mesh resolution on the dissolution process during phase- 2 is studied using the four meshes described in Section 3.4. This mesh sensitivity study is performed with the standard $k-\varepsilon$ turbulence model with EWT and full buoyancy. In all
Time $=4300 \mathrm{~s}$

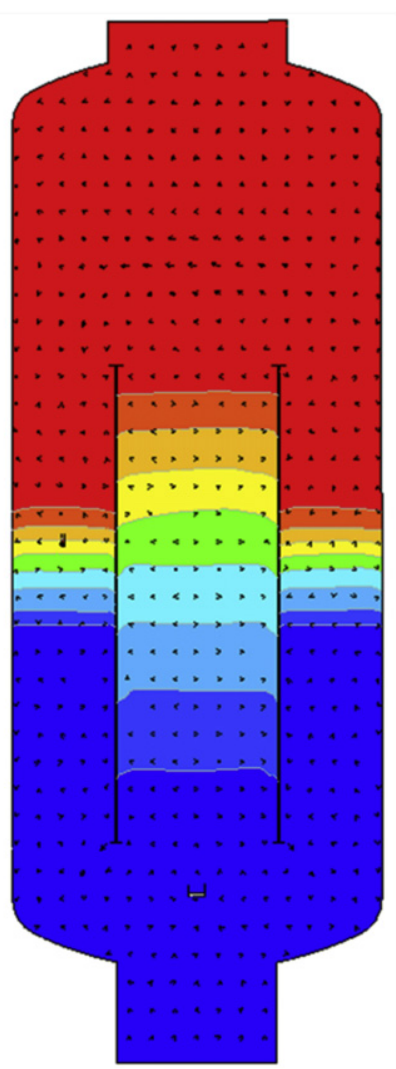

Time $=4500 \mathrm{~s}$

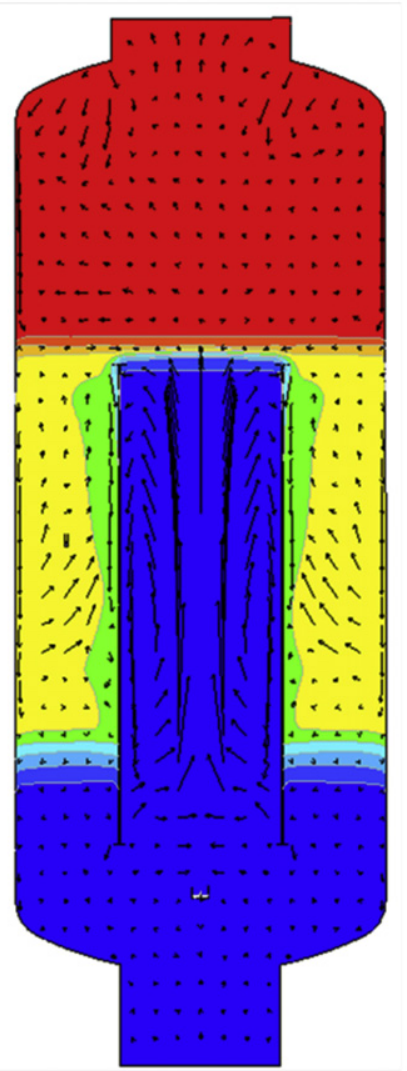

Time $=5000 \mathrm{~s}$

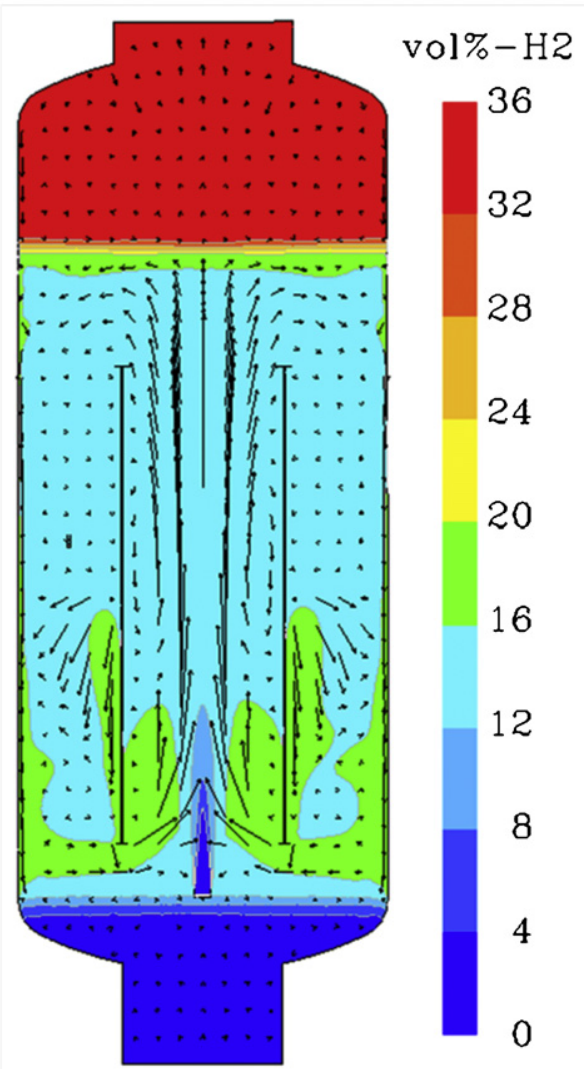

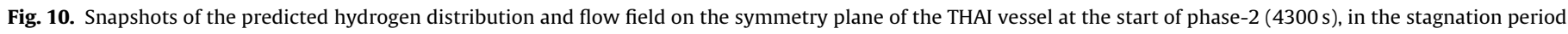
$(4500 \mathrm{~s})$ and in the natural circulation period $(5000 \mathrm{~s})$. 


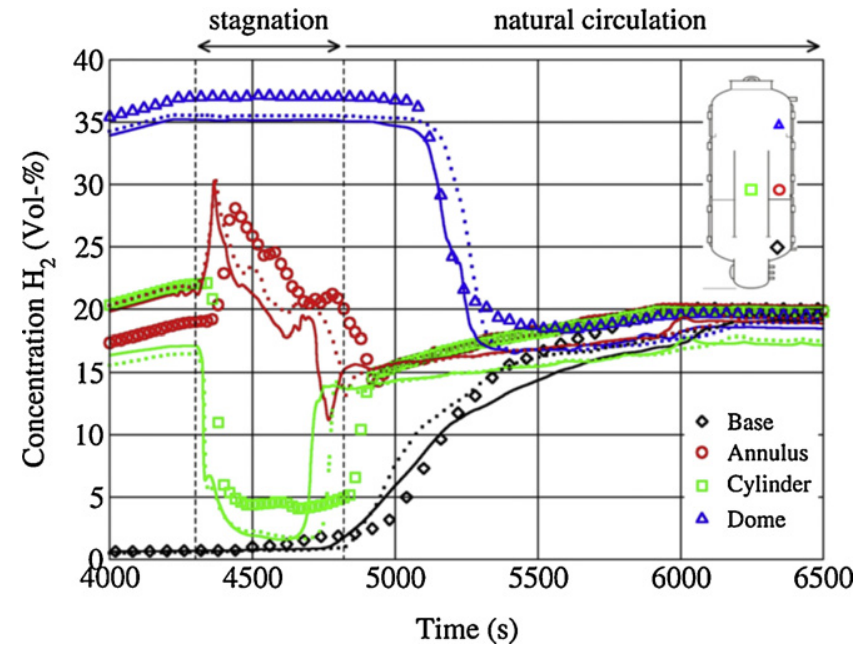

Fig. 11. Comparison of the measured hydrogen concentrations in the vessel (symbols) with those computed on the $y^{+}=1$ mesh (solid lines) and standard mesh (dotted lines).

calculations the convergence was in the order of $10^{-5}$ for flow and species, and in the order of $10^{-9}$ for energy.

The $y^{+}=1$ and standard mesh have the same mesh resolution in the bulk and a different mesh resolution near the wall. To assess the effect of near-wall treatment, the results obtained on the $y^{+}=1$ and standard mesh are compared qualitatively in Fig. 11 and quantitatively in Table 5. Fig. 11 shows again the development of the hydrogen concentration over time at the four locations in the vessels' base, annulus, cylinder and dome compartment. Table 5 shows values of pressure, hydrogen concentration, mass conservation and the time of onset of natural circulation for the different meshes. The results obtained on the standard mesh with wall functions and on the $y+=1$ mesh with the two-layer model are very similar. The overall mixing process during phase- 2 is slightly slower for the standard mesh. Nevertheless, the results agree well and are both close to the experimental results, meaning that wall functions are applicable to model the THAI HM-2 test.

The standard, coarse and fine mesh have the same mesh resolution near the wall and a different mesh resolution in the bulk (see Table 3). The effect of bulk mesh resolution can be observed in Fig. 12 and Table 5, where the results obtained on the

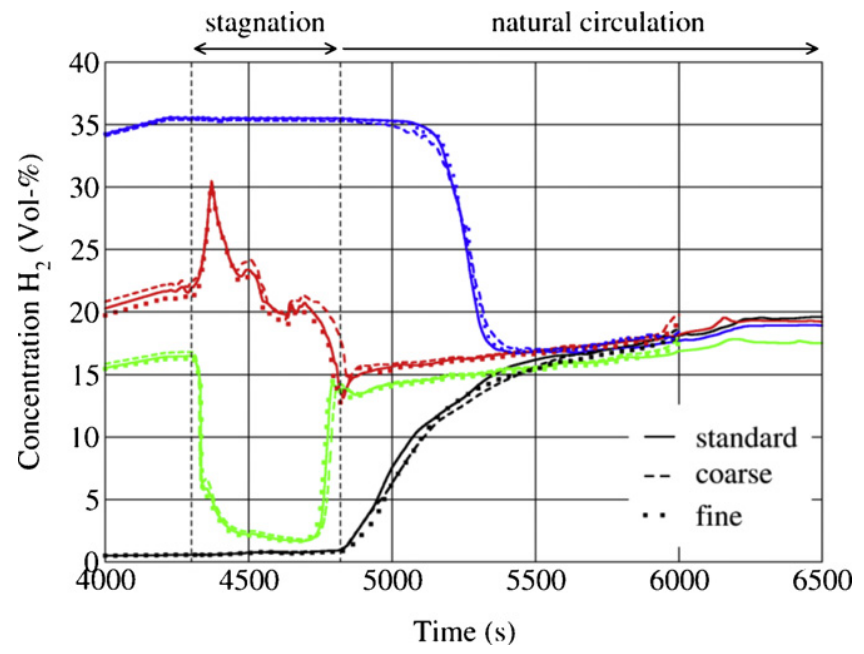

Fig. 12. Comparison of the hydrogen concentrations in the vessel as computed on the standard mesh (solid line), coarse mesh (broken line), and fine mesh (dotted line).
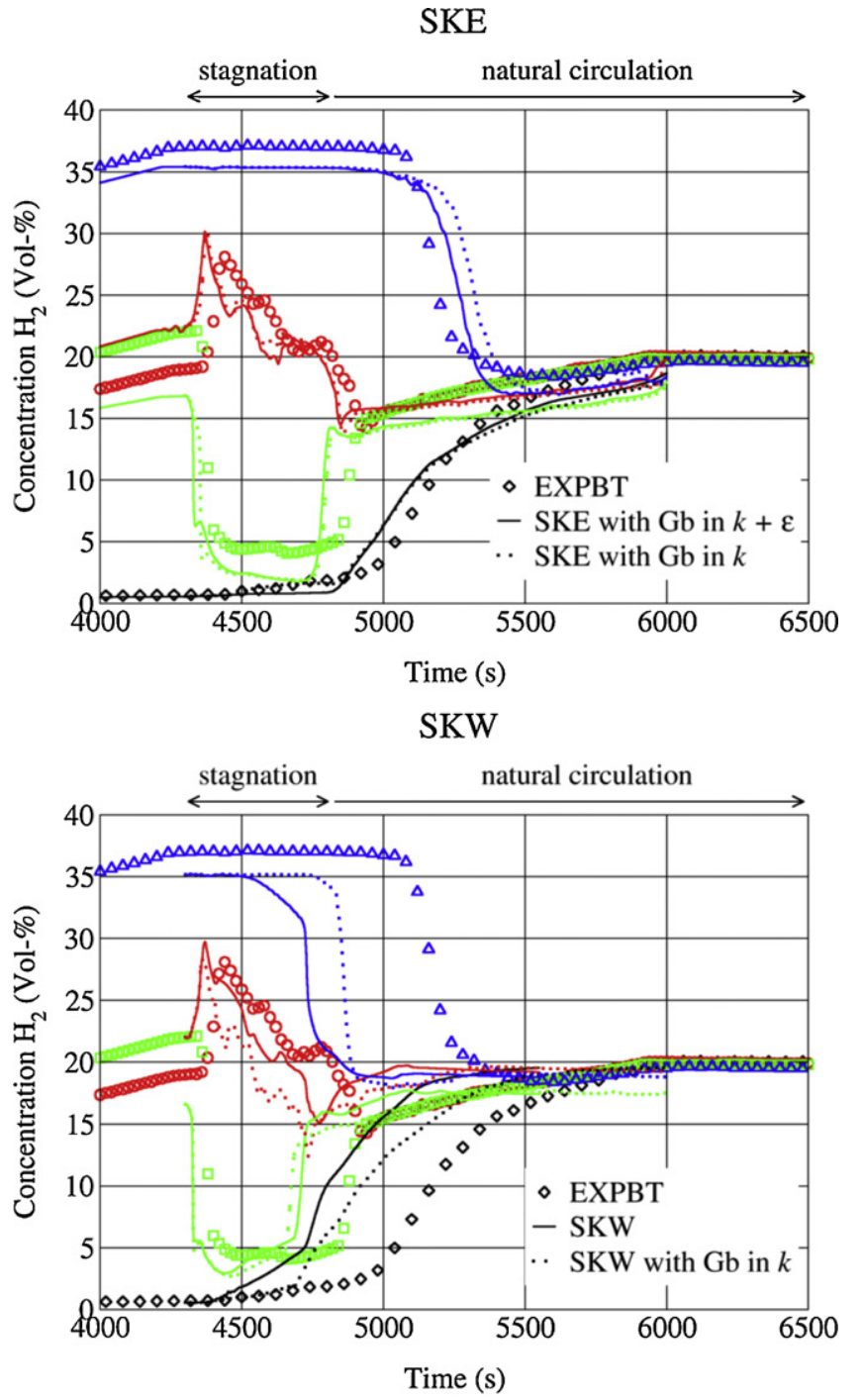

SSTKW

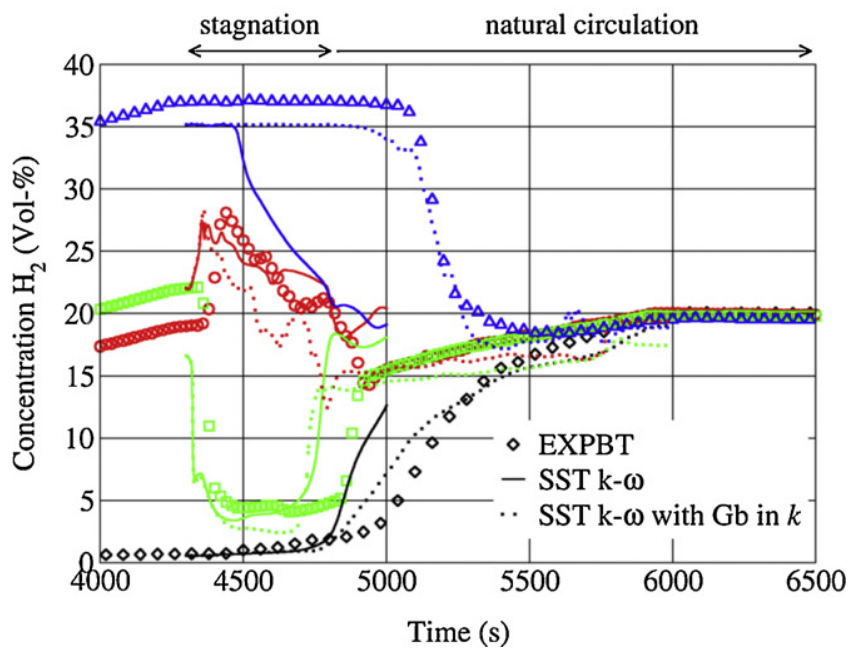

Fig. 13. Hydrogen concentrations in the vessel atmosphere during phase- 2 as measured (EXPBT) and computed with different turbulence models and buoyancy terms. 

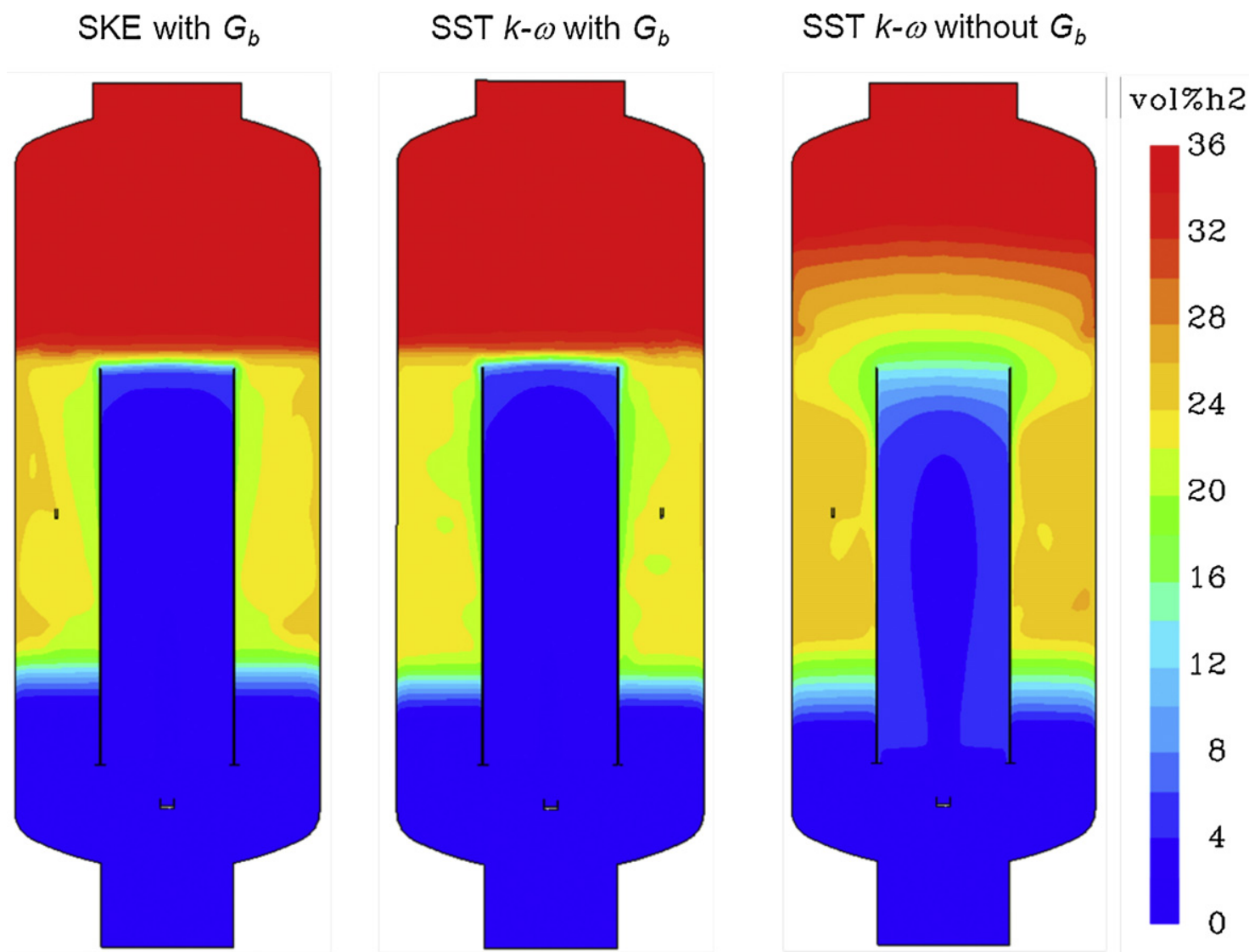

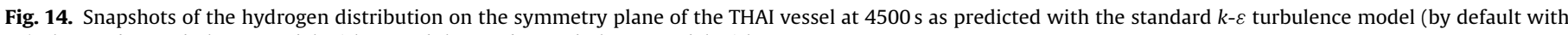
$G_{b}$ ), the SST $k-\omega$ turbulence model with $G_{b}$ and the SST $k-\omega$ turbulence model without $G_{b}$.

standard, coarse and fine mesh are compared. The differences are small, which shows that a cell size of $45 \mathrm{~mm} \times 75 \mathrm{~mm}$ in the bulk is sufficiently small to model the THAI HM-2 test.

\subsection{The effect of turbulence model}

In this section, the effects of the turbulence model and buoyancy terms in the turbulence models on the prediction of the dissolution process during phase- 2 are studied for the settings given in Table 4. All analyses are performed on the coarse mesh. Apart from the turbulence settings, the analyses are performed with the same settings. In order to assure identical starting conditions for phase2 , all calculations start at $4300 \mathrm{~s}$ on the phase- 1 solution obtained with the standard $k-\varepsilon$ model with full buoyancy on the coarse mesh (case 1 in Table 4).

Fig. 13 compares the computed hydrogen concentrations in the vessel atmosphere during phase- 2 for the different turbulence settings listed in Table 4 . The stagnation period and onset of natural circulation is predicted reasonably well in all calculations. However, compared to the experiment, the dissolution of the hydrogen rich cloud sets in too early and the dissolution rate is higher for the standard $k-\omega$ and SST $k-\omega$ models without $G_{b}$ in $k$, as implemented by default in FLUENT. The prediction of both these $k-\omega$ models improves when $G_{b}$ is included in the transport equation of the turbulent kinetic energy $k$. Although the standard $k-\omega$ model with $G_{b}$ in $k$ still predicts a slightly higher dissolution rate of the hydrogen rich cloud compared to the experiment, the results of the SST $k-\omega$ model with $G_{b}$ agree well with experiment and are very similar to the results of the standard $k-\varepsilon$ model with $G_{b}$. Apparently, the generation (or dissipation) of turbulent kinetic energy by buoyancy has a large effect on the stability and dissolution process of the stratified hydrogen-rich layer. This influence of buoyancy can be understood as will be explained in the next paragraph. Including the buoyancy effect in the turbulent dissipation rate $\varepsilon$ of the standard $k-\varepsilon$ model shows no significant influence.

Fig. 14 shows the hydrogen distribution on the symmetry plane at $4500 \mathrm{~s}$ as calculated with the standard $k-\varepsilon$ model with $G_{b}$ in $k$ and $\varepsilon$, the SST $k-\omega$ model with $G_{b}$ in $k$ and the SST $k-\omega$ model without $G_{b}$. At 4500 s the HM-2 test is in the stagnation period of phase- 2 . In the experiment, the injected steam flows into the inner cylinder during the stagnation phase without affecting the hydrogen concentration in the dome area above the inner cylinder. As shown in Figs. 13 and 14, this is only true for the calculation with the standard $k-\varepsilon$ turbulence model and the SST $k-\omega$ model with $G_{b}$. The snapshots in Fig. 14 show that in the calculation of the SST $k-\omega$ model without $G_{b}$, some of the steam flowed out of the inner cylinder and mixed with the hydrogen rich cloud at 4500 s. This proves that it is important to take the effect of buoyancy into account in the turbulence model by including the $G_{b}$ term. Turbulence is suppressed by buoyancy $\left(G_{b}<0\right)$ near the stratified hydrogen rich layer. Without this damping effect, the stratified layer above the inner cylinder will be dissolved faster by turbulent mixing. Consequently, the turbulence models without $G_{b}$ do not correctly capture the dissolution process during phase-2.

\section{Conclusions}

In the present work, the containment model of NRG is further validated in the context of hydrogen distribution in a containment using the THAI HM-2 test. For this test, the characteristic phenomena are the development and dissolution of a hydrogenrich gas layer in the upper part of the THAI containment by a 
buoyant plume. These phenomena are predicted well by NRG's containment model developed in FLUENT. Additional sensitivity analyses on mesh resolution, near-wall treatment, and turbulence model and turbulence model settings, showed the following:

- A cell size of $45 \mathrm{~mm} \times 75 \mathrm{~mm}$ in the bulk is sufficiently small to model the hydrogen distribution and dissolution processes in the THAI HM-2 test with CFD.

- Good CFD results are obtained when wall functions are applied to model the flow in the near wall region for the HM-2 test.

- The effect of buoyancy on the turbulent kinetic energy $k$ is taken into account by default in the $k-\varepsilon$ turbulence models of FLUENT. This effect on $k$ is not incorporated by default in the $k-\omega$ models of FLUENT, but it can be incorporated by user coding.

- The prediction of the dissolution process during phase- 2 of the HM-2 test improves when the effect of buoyancy on $k$ is included in the turbulence models. Without this effect the CFD model predicts a too early start of the dissolution, as well as a too high dissolution rate of the stratified hydrogen-rich layer.

- The results of the standard $k-\varepsilon$ model and SST $k-\omega$ turbulence model with the effect of buoyancy on $k$ included are very similar and agree well with experiment, whereas the standard $k-\omega$ turbulence model with the effect of buoyancy on $k$ included still predicts a higher dissolution rate.

- The effect of buoyancy on the turbulent dissipation rate $\varepsilon$ can be included in the $k-\varepsilon$ models of FLUENT by activating the "full buoyancy" option. The buoyancy effect on $\varepsilon$ is subject to discussion in literature. For the HM-2 test, there was no significant impact on the results obtained with CFD.

These findings, combined with the extensive model description as presented in this paper may serve others in improving the predictive quality of CFD for containment analyses. Furthermore, these findings and settings can develop into a set of best practice guidelines for the application of CFD for containment analyses. To this end, more experimental tests should be analyzed in the future to confirm the general applicability of the presented containment model and model settings. Work in this area is currently ongoing at NRG.

\section{Acknowledgments}

The authors thank all signatories and participants to the OECDNEA THAI project. Special thanks are expressed to the people involved in the experiments and documentation. In addition, the authors gratefully acknowledge the funding provided by the Department of Nuclear Safety, Security and Safeguards (KFD) that is presently part of the Dutch Ministry of Infrastructure and Environment.

\section{References}

Allelein, H.J., et al., 2007. International Standard Problem ISP-47 on Containment Thermalhydraulics, Final Report. NEA/CSNI, OECD, Paris.

Ambrosini, W., et al., 2007. Results of the SARNET Condensation Benchmark No. 0. DIMNP 006(2007). University of Pisa.

Andreani, M., Paladino, D., 2010. Simulation of gas mixing and transport in a multicompartment geometry using the GOTHIC containment code and relatively coarse meshes. Nucl. Eng. Des. 240, 1506-1527.

Bentaib, A., Bleyer, A., 2011. ASTEC validation on OECD/THAI HM-2. In: Proceedings of ICAPP'11, Nice, France, May 2-5, Paper 11258.

ERCOFTAC, 2000. Best Practice Guidelines, www.ercoftac.org.

Fischer, K., 2004. International Standard Problem ISP-47 on Containment ThermalHydraulics, Step 2: ThAI, Volume 1: Specification Report. Becker Technologies GmbH, Eschborn, Report No. BF-R 70031-1 Rev. 4.

FLUENT 6.3 User's Guide, 2006. FLUENT Inc.

GASFLOW, 2011. http://hycodes.net/gasflow.

Gupta, S., Kanzleiter, T., Fischer, K., Langer, G., Poss, G., 2010. Interaction of a stratified light gas layer with a buoyant jet in containment: hydrogen/helium material scaling. In: Proceedings of ICAPP'10, San Diego, CA, USA, June 13-17, Paper 10205.

Heitsch, M., Baraldi, D., Wilkening, H., 2010. Simulation of containment jet flows including condensation. Nucl. Eng. Des. 240, 2176-2184.

Houkema, M., Siccama, N.B., Lycklama à Nijeholt, J.A., Komen, E.M.J., 2008. Validation of the CFX4 CFD code for containment thermal-hydraulics. Nucl. Eng. Des. 238, 590-599.

Kanzleiter, T., Fischer, K., 2008. Quick Look Report Helium/Hydrogen Material Scaling Test HM-2. Becker Technologies GmbH, Eschborn, Germany, OECD-NEA THAI Project Report No. 1501326 - HM-2 QLR Rev. 3.

Kudriakov, S., Dabbene, F., Studer, E., Beccantini, A., Magnaud, J.P., Paillère, H., Bentaib, A., Bleyer, A., Malet, J., Porcheron, E., Caroli, C., 2008. The TONUS CFD code for hydrogen risk analysis, physical models, numerical schemes and validation matrix. Nucl. Eng. Des. 238, 551-565.

Lemmon, E.W., Huber, M.L., McLinden, M.O., 2007. NIST Standard Reference Database 23: Reference Fluid Thermodynamic and Transport PropertiesREFPROP, Version 8. 0. National Institute of Standards and Technology, Standard Reference Data Program, Gaithersburg.

Menter, F.R., 1994. Two-equation Eddy-viscosity turbulence models for engineering applications. AIAA J. 32 (8), 1598.

Prabhudharwadkar, D.M., Iyer, K.N., Mohan, N., Bajaj, S.S., Markandeya, S.G., 2011. Simulation of hydrogen distribution in an Indian nuclear reactor containment. Nucl. Eng. Des. 241, 832-842.

Royl, P., Travis, J.R., Breitung, W., Kim, J., Kanzleiter, T., Schwarz, S., 2009. GASFLOW analysis of steam/hydrogen mixing with nitrogen in the OECD-NEA THAI HM-2 benchmark. In: Proc. of NURETH-13, Kanazawa, Japan, September 27-October 2, Paper N13P1412.

Schwarz, S., Fischer, K., Bentaib, A., Burkhardt, J., Lee, J.-J., Duspiva, J., Visser, D., Kyttala, J., Royl, P., Kim, J., Kostka, P., Liang, R., 2010. Benchmark on hydrogen distribution in a containment based on the OECD-NEA THAI HM-2 experiment. Nucl. Technol. 175, 594-603.

SOAR, 1999. SOAR on Containment Thermalhydraulics and Hydrogen Distribution, NEA/CSNI/R(1999)16. 